\title{
One Global Map but Different Worlds: Worldwide Survey of Human Access to
}

\section{Basic Utilities}

\section{Florin-Constantin Mihai}

\section{Human Ecology}

An Interdisciplinary Journal

ISSN 0300-7839

Hum Ecol

DOI 10.1007/s10745-017-9904-7

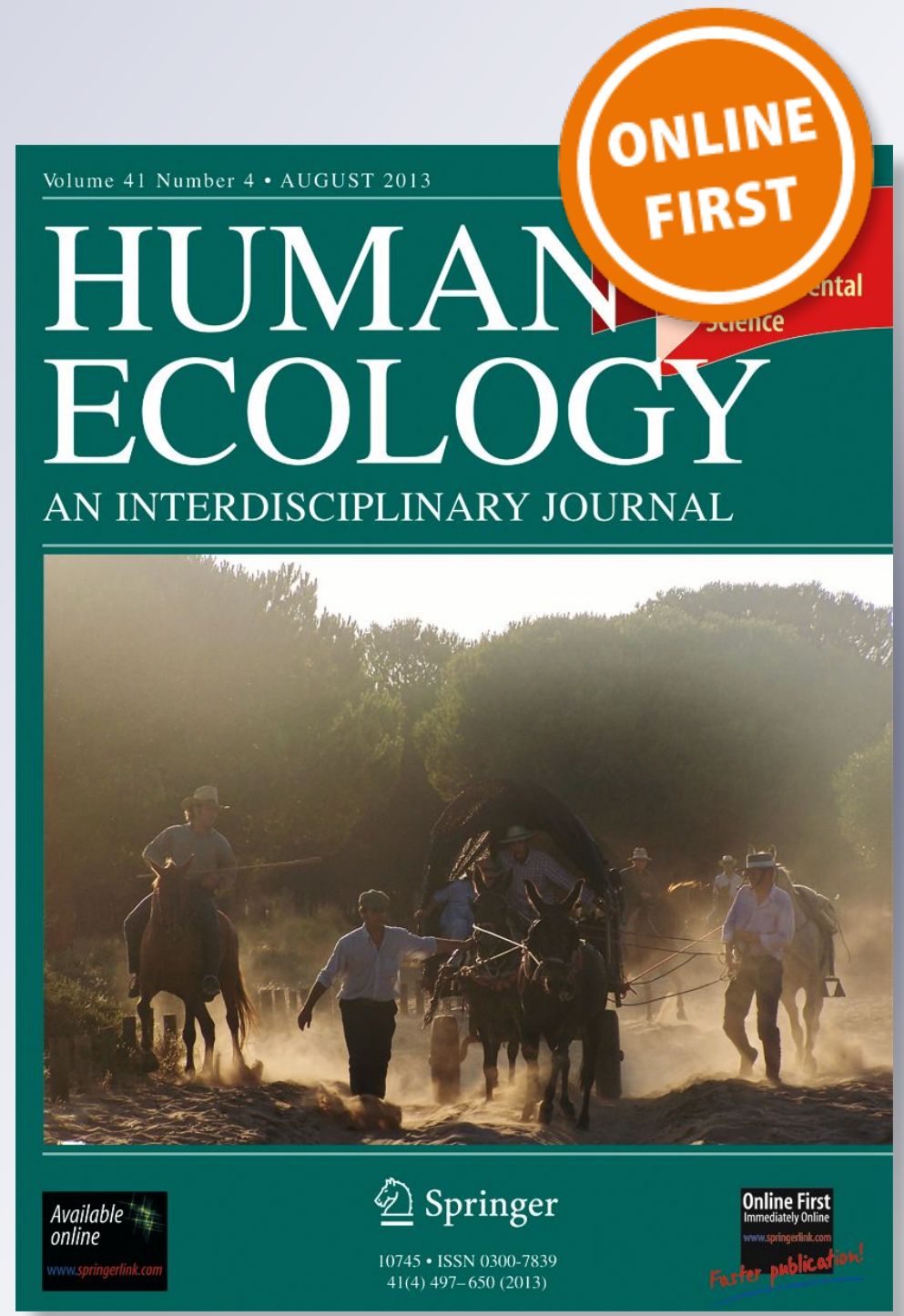

留 Springer 
Your article is protected by copyright and all rights are held exclusively by Springer Science +Business Media New York. This e-offprint is for personal use only and shall not be selfarchived in electronic repositories. If you wish to self-archive your article, please use the accepted manuscript version for posting on your own website. You may further deposit the accepted manuscript version in any repository, provided it is only made publicly available 12 months after official publication or later and provided acknowledgement is given to the original source of publication and a link is inserted to the published article on Springer's website. The link must be accompanied by the following text: "The final publication is available at link.springer.com". 


\title{
One Global Map but Different Worlds: Worldwide Survey of Human Access to Basic Utilities
}

\author{
Florin-Constantin Mihai $^{1}$
}

(C) Springer Science+Business Media New York 2017

\section{Introduction}

There are major disparities among and within continents, countries, regions, cities, and rural municipalities in access to sources of safe drinking water, sanitation facilities, regular waste collection services, electricity, and other critical amenities. There are both conguities and disparities overlapping along shared geographical and socioeconomic lines as well as historical antecedents. The global report of WHO and UNICEF's Joint Monitoring Programme for Water Supply and Sanitation (JMP) reveals some startling inequalities, particularly in the water, sanitation and hygiene sector (WASH), where 2.5 billion people have no access to an" improved" sanitation facility and 1 billion people still practice open-air defecation (WHO-UNICEF 2014). The Global Waste Management Outlook (UNEP-GWMO 2015) estimates that 2 billion people lack solid waste collection services and 3 billion of people lack access to controlled waste disposal facilities. More than half of world population (54\%) in 2014 lives in urban areas (UN 2014). Population growth and rapid urbanization in developing countries continue to offer serious challenges regarding basic public utilities. Rural to urban migration often means that urban municipalities are overwhelmed and consequently provide poor quality services. Overall, $70 \%$ of those without access to improved sanitation

Electronic supplementary material The online version of this article (doi:10.1007/s10745-017-9904-7) contains supplementary material, which is available to authorized users.

Florin-Constantin Mihai

mihai.florinconstantin@gmail.com

1 Department of Research, Faculty of Geography and Geology, "Alexandru Ioan Cuza" University, Carol I Blvd, Nr. 20 A, Iasi, Romania facilities live in rural areas (WHO-UNICEFF 2014). This paper provides a holistic or descriptive approach that combines WASH data sets with other statistics on basic utilities such as waste collection coverage and access to electricity. These are broken down for urban (U) and rural (R) populations and are related to key demographic features in a geographical context at global scale.

I examine population access to basic utilities at the national level ( $\mathrm{T}$ or total) and broken down for urban and rural populations $(U, R)$ where these data are available. Sixteen indicators with global coverage are selected for the cluster analysis. Each variable is expressed in percentage values. Most of the aggregate data are provided by international organizations, except for the waste collection coverage (T, U, R). I relied on literature review and reasonable assumptions to compute such data.

\section{Selected Basic Utilities - Data Provided by International Organizations}

\section{Improved drinking water sources available for total, urban and rural population $(T, \mathbf{U}, \mathbf{R})$}

According to WHO/UNICEEF Joint Monitoring Programme (JPM) for Water Supply and Sanitation an "improved drinking-water source is one that, by the nature of its construction, or through active intervention adequately protects the source from outside contamination, particularly faecal matter." The following drinking water sources are considered by JMP: piped water into dwelling, piped water to yard/plot, public tap or standpipe, tubewell or borehole, protected dug well, protected spring, rainwater. ${ }^{1}$

\footnotetext{
${ }^{1}$ See more: http://www.wssinfo.org/definitions-methods/watsan-categories.
} 


\section{Improved Sanitation Facilities (T, U, R)}

WHO/UNICEF defines an "improved sanitation facility as the one that hygienically separates human excreta from human contact." Following sanitation facilities are considered improved by JMP: flush toilet, piped sewer system, septic tank, flush/pour flush to pit latrine, ventilated improved pit latrine (VIP), pit latrine with slab, composting toilet.

\section{Access to Electricity (T)}

This indicator refers to the percentage of total population connected to electricity. The data source is provided by the World Bank via the Global Electrification database (http://data. worldbank.org/indicator/EG.ELC.ACCS.ZS). The electrification rate for urban and rural, i.e., the percentage of households connected to electricity, and assumptions about an average household size are used by International Energy Agency (IEA) (2014) in order to determine access rates as a percentage of the population.

\section{Population Using Solid Fuels as an Energy Resource (T)}

This indicator is part of the Millenium Development Goals (MDG) monitored by UN Statistics which outlines "the percentage of the population that relies on solid fuels as the primary source of domestic energy for cooking and heating." According to the UN, solid fuels include biomass fuels, such as wood, charcoal, crops or other agricultural waste, dung, shrubs and straw, and coal. (http://mdgs.un. org/unsd/mdg/Metadata.aspx? IndicatorId=29).

\section{Assessment of Global Waste Collection Services (WCS)}

The share of the population covered by regular municipal waste collection services is the most difficult to measure and to interpret at global scale due to multiple sources of information and documents, scarcity of reliable data for low and middle-income countries, and that some data are provided only for urban areas and total coverage must be extrapolated based on demographic data. Also, there it may not be clear whether the data refer to a capital city or all urban areas, and there are various definitions of municipal wastes/household/domestic waste in different countries. Data sources used in order to compute the waste collection coverage vary across countries as do reliable estimates of waste collected out of the total generated (collection efficiency). Therefore, data inconsistencies are inherent at the global scale. A comparative analysis is performed for data provided by organizations, national statistics, "gray" and peer-reviewed literature, and websites in order to improve data as much as possible (see Supplementary material 1 and 2).

\section{Urban Population Served (WCS_U)}

Data concerning waste collection coverage rates in urban areas are available in the literature and technical reports, but their quality varies among sources. Poorly updated data are the norm in the case of developing countries in Africa and Asia. Some data are available only for one city. Previous studies used such data in order to extend this rate to all urban areas due to the poor availability of data, but this should be avoided because within a given country major disparities may exist between large, mid-size, and small cities. In such cases, the means are calculated to represent the WCS rates for the urban population (see Supplementary material 1 and 2).

\section{Rural Population Served (WCS_R)}

For low-income countries I assume no formal waste collection is provided in rural areas $(0.5 \%)$ except in the cases where such data are available in the literature or other sources (see Supplementary material 1 and 2).

\section{Demographic Features}

Urban population (\%); slum of urban population (\%), population in the largest city (\%) as part of UN-World Urbanization Prospects (see Supplementary material 1 and 2).

\section{Results}

The average values (arithmetic means) of this classification (179 spatial units) outline several features: $58 \%$ of the population of these 179 states and territories lives in urban areas, of which $21.61 \%$ are in slum areas with poor access to basic utilities. The largest cities (often the country's capital) account for $33.39 \%$ of the urban population. In all 179 states and territories $59.49 \%$ of inhabitants are covered by waste collection services and $74 \%$ have sanitation facilities, key elements for decent public health. The population is better served by drinking water facilities $(88 \%)$ and electricity (79\%), but $32 \%$ are still dependent on solid fuels for basic needs (cooking, heating, etc.). Urban-rural disparities are significant in the case of low and middle-income countries. At the global scale, $76.16 \%$ of the urban population are connected to regular waste collection services and $79.68 \%$ to improved sanitation facilities, but these indicators drop to $43 \%$ and $68.4 \%$ in rural areas. Improved drinking water sources 


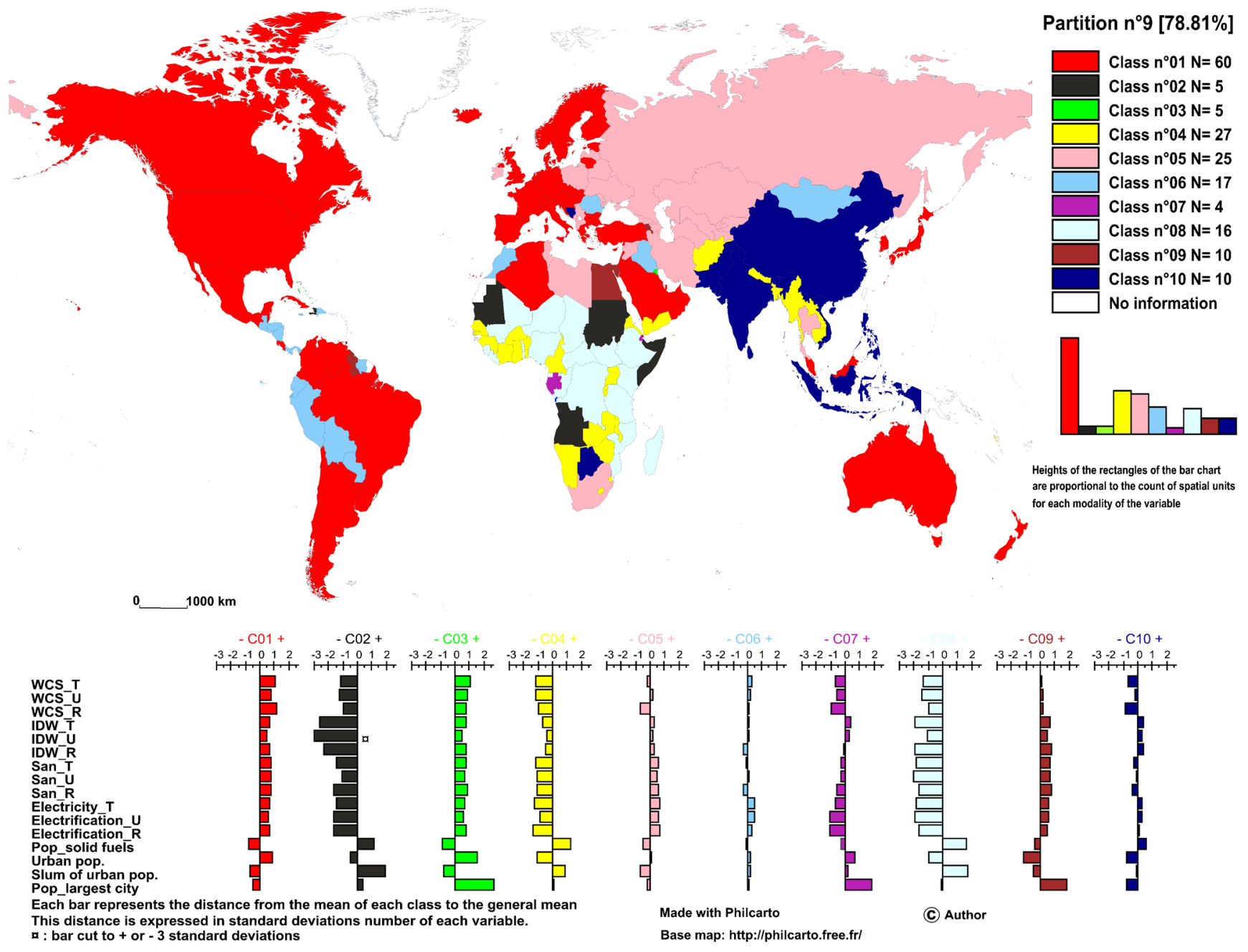

Fig. 1 Population access to basic utilities: global disparities (about here)

and electrification rate have higher coverage rates in urban areas. Hierarchical cluster analysis outlines following 10 typologies (Fig. 1).

- Class 1: This class includes most spatial units (60) across America, Europe, Asia, and Oceania where the population is well served ( $>94 \%)$ by each basic facility above the global trend (central bar).

- Class 2: Severe problems regarding access to improved drinking water facilities (48.7\%) with significant impacts on public health. The other basic utilities are poorly available even for urban populations $(<50 \%)$, which are mostly concentrated in the rudimentary slum areas $(75 \%$ - the highest level across all classes).

- Class 3: This class groups city-states, island, or small high-income countries (e.g., Singapore, San Marino, Kuwait, Hong Kong, the Bahamas) where the majority of the population lives in the capital city where they have almost full access to all basic utilities.
- Class 4: The second largest class, with 27 countries, has low coverage of WCS (mean 17\%). Less than half of the urban population has access to regular WCS and such services are lacking entirely in rural areas. Population access to drinking water source, sanitation facilities, and electricity is below the global average. Almost $66 \%$ of this population is using solid fuels for domestic purposes.

- Class 5: The third largest class (25) where most basic utilities are provided above the general average (central bar) for over $85 \%$ of the population (total, urban or rural) excluding WCS, particularly in rural areas.

- Class 6: This class has values close to the general average (central bar), showing similarities with Class 5. Sanitation facilities have poorer coverage rates with significant gaps between urban (81\%) and rural populations (58.4\%).

- Class 7: This class includes urbanized developing countries $(73.85 \%)$ where the majority of the urban population lives in the capital city (74\%), with $25 \%$ in slum areas (Gabon, Djibouti,) with poorer access to electricity. 
Rural areas often have no WCS and sanitation facilities are provided for less than $50 \%$ of the population.

- Class 8: This class represents one of the worst-case scenarios. Waste collection services are lacking or poorly provided even in urban areas (37.31\%). The populations of these countries have the poorest access to waste collection services $(12.72 \%)$ and improved sanitation facilities $(16.33 \%)$ Households are dependent on solid waste fuels $(90.8 \%)$ and most have restricted access to electricity $(20.15 \%)$ across all classes.

- Class 9: This class has significant values above the central bar for total urban and rural population. The majority of inhabitants ( $>89 \%$ ) have access to improved drinking water, sanitation and electricity services despite the fact that the urban population is only $30 \%$ (lowest of all classes) compared with Class $1(78.56 \%)$ or Class 7 (73.85\%).

- Class 10: This class has poor waste collection coverage rates with major disparities between urban (71.7\%) and rural populations $(6.8 \%)$. Such discrepancies are also found for sanitation facilities. More than half of the population uses solid fuels as a domestic energy source and lives in rural areas.

See the Supplementary material 1 and 2 for a further examination of data processing and limitations of this study.

\section{Discussion}

Sustainable Development Goals (SDGs) have a key-target to ensure access for all to adequate, safe and affordable housing and basic services by 2030. Furthermore, UNEP-GMWO ( 2015) establish as priorities the extension of the municipal solid waste collection to $100 \%$ of the urban population, to eliminate uncontrolled dumping and open burning by 2020 . Such targets are difficult to achieve in the current context. My estimates show that approximately 2.8 billion people lack waste collection services, 0.9 billion in urban areas and 1.9 billion in rural areas. This reveals a worse situation at the global scale than the 2 billion of people reported by UNEP GMWO ( 2015). This result may be explained by the fact that this study integrates more spatial units with specific data broken down for the total, urban and rural population. Despite the fact that MDGs ignore the role of the municipal waste management sector as a key indicator for sustainable development, Gonzenbach and Coad (2007) highlight the significant progress which a sound solid waste management can produce towards these goals. The waste management sector regularly covers large and mid-size cities, but small urban areas and rural regions are often neglected in the provision of WCS, improved sanitation and drinking water facilities, and they have poor connections to electricity. The disparities between urban and rural settlements are significant in the large and well-populated countries (C5\&C10). Rural migration of poor people to the slum areas of mega-cities amplifies this environmental crisis. Across the world, populations living in urban slums are highly predisposed to pollution due to the lack or rudimentary waste and sanitation facilities, as shown by classes C2, C4 \& C8. The solid waste services for African countries are the most decentralized and fragmented of all basic services involving public, private, informal, and civil society sectors (UCGL 2013). Open defecation and waste dumping on surrounding areas are common practices among poor populations.

The populations of high-income countries do not use solid fuels as a domestic energy source compared to lower income countries where these are crucial for daily life (C2, C4, C8 \& C10). Solid fuels are used by more than $90 \%$ of inhabitants in several African countries, Asia (Bangladesh, Laos) and Haiti. Poor energy access translates into poverty through poor economic performance. In this context waste incineration and landfill gas may be alternative energy sources for African urban areas (Scarlat et al. 2015).

Frequently, a high share of the urban population of a country may have a positive effect on waste collection coverage or sanitation facilities (C01 \&C03), but in developing countries, major disparities may appear from one country/city to another. Sanitation facilities are related to waste management systems and such disparities must be examined in the field, but frequently WASH studies ignore this aspect. The populations which live in informal settlements are often excluded from such statistics so that it is difficult to gather accurate data. Despite the fact that low-income countries generate smaller amounts of household waste, they also have low collection rates, around $41 \%$, and there are enormous variations in service across and within cities, especially between slum and non-slum areas (UCLG 2013). Rural populations in Africa have serious challenges to get access to safe drinking water sources, which frequently are inadequate, unreliable, and inaccessible (Alhassan and Kwakwa 2014). Countries from classes C2 and C8 (21), which include only one country outside Africa - Haiti, are most deprived of this basic need for survival. Pullan et al. (2014) performed a critical analysis with proper estimations concerning population access to drinking and sanitation facilities at national and subnational levels for sub-Saharan Africa, outlining the regional disparities within countries. Poor Asian countries and emergent economies (included in $\mathrm{C} 4$ ) have outdated sanitation and waste management infrastructures and the improved drinking water facilities are below the global average. The natural hazards such as earthquakes (Haiti, Nepal) or floods (Bangladesh, India) damage already poor infrastructures. Post or current conflict areas and political disputes weaken public or private investments to improve the basic utilities for underserved populations (Afghanistan, Iraq, Syria, Libya). Nor do former soviet countries across Central and Eastern Europe (included in C5 \& C6) have full coverage of improved sanitation or WCS. New EU members have many challenges to fulfill the EU requirements 
in their sanitation and waste management sectors (World Bank 2011). After the breakup of the Soviet Union, the Eurasian countries were left with sufficiently developed water supplies and sanitation, district heating, and urban public transport, but solid waste management received less attention (UCGL 2013). Based on this research, I estimate that waste collection coverage is under $50 \%$ of total population in 76 countries, and under $50 \%$ of the rural population in 105 countries.

Poor coverage rates of improved sanitation facilities $(<50 \%)$ are found in 49 countries in the case of the total population, $33 \%$ of urban and $60 \%$ of the rural population. Population access to electricity is under $50 \%$ in 45 countries, and electrification rates are under $50 \%$ in 55 countries. Despite the fact improved drinking water facilities have better coverage at the global scale, there are 18 countries where half or less of the rural population has access to such facilities. Frequently, rural waste management almost is non-existent in developing countries, or official data are not available. At least half of the population from 66 countries depend on solid fuels for daily life. Some countries have coverage rates below $10 \%$ for all three basic indicators: WCS, improved sanitation facilities (T), and electricity (Malawi, Niger, South Sudan). The map (see above) highlights that worst scenario cases regarding population access to basic utilities are encountered across African countries. Restricted access to electricity and poor sanitation facilities combined with the lack of or rudimentary WCS in rural and crowded slum areas make living conditions barely supportable in developing countries. The poor urban population is concentrated in slum areas $(>40 \%)$ in 49 countries particularly in Africa and Asia.

\section{Conclusions}

The map and hierarchical cluster analysis reveal major disparities across the globe concerning populations' access to basic utilities. There are three classes (C2, C8, \& C04), which include 48 countries (40-Africa, 7-Asia, 1-Oceania) with severe issues in providing all basic utilities representing the worst scenario cases. In Classes 1 and 3 (65 spatial units) these basic utilities are commonplace for almost all. Furthermore, Classes 7 and 10 (14 spatial units) have better coverage of improved drinking water facilities, but poor sanitation and WCS. Class 5 (25 spatial units) includes countries that have serious difficulties in providing WCS and improved sanitation facilities, particularly in rural areas. The global trend with values close to the general average is reflected by Class 6 (17 spatial units), which partially covers the population with basic utilities and where major improvements are needed for small cities and rural areas. Coverage of the total population with basic utilities is afforded only by 65 countries out of 179 (36.3\%) included in the cluster analysis. Such geographical inequalities are also amplified by demographic features, such as the frequent concentration of the populations of developing countries in slum areas and larger cities due to internal migration, which it makes more difficult to improve basic utilities. A high percentage of poor people depend on solid fuels as a domestic energy source, and the access to electricity is very limited especially in the poorest countries. Governments of low and middle-income countries fail frequently to provide for the basic needs of their populations.

\section{Compliance with Ethical Standards}

Funding This work is the result of fully independent research without funding.

Conflict of Interest The author declares that he has no conflict of interest.

\section{References}

Alhassan H., and Kwakwa P. A. (2014). When water is scarce: The perception of water quality and effects on the vulnerable. Journal of Water, Sanitation and Hygiene for Development. 04-1: 43-50.

Gonzenbach B and Coad A. 2007. Solid waste management and the millennium development goals. CWG Publication Series No 3. ISBN 3-908156-11-4 Available at: http://www.ircwash. org/sites/default/files/Gonzenbach-2007-Solid.pdf (Accessed 9 October 2015)

International Energy Agency (IEA) (2014). World Energy Outlook Electricity Access Database. https://www.iea.org/publications/ freepublications/publication/WEIO2014.pdf

Pullan R. L., Freeman M. C., Gething P. W., and Brooker S. J. (2014). Geographical inequalities in use of improved drinking water Supply and sanitation across sub- Saharan Africa: Mapping and spatial analysis of cross-sectional survey data. PLoS Med 11(4): e1001626. doi:10.1371/journal.pmed.1001626.

Scarlat N., Motola V., Dallemand J. F., Monforti-Ferrario F., and Mofor L. (2015). Evaluation of energy potential of municipal solid waste from African urban areas. Renewable and Sustainable Energy Reviews 50: $1269-1286$.

UNEP. 2015. Global Waste Management Outlook (GWMO)

United Cities and Local Governments (UCLG) 2013 Basic services for all in an urbanizing world, Third Global Report of United Cities and Local Governments on Local Democracy and Decentralization GOLD III Executive Summary www.uclg.org

United Nations. Department of Economic and Social Affairs, Population Division. 2014. World Urbanization Prospects: The 2014 Revision, Highlights (ST/ESA/SER.A/352).

WHO/UNICEF Joint Monitoring Programme for Water Supply and Sanitation (2014). Progress on sanitation and drinking-water: 2014 update, World Health Organization, Geneva.

World Bank. 2011. Solid Waste Management in Bulgaria, Croatia, Poland, and Romania: A Cross-Country Analysis of Sector Challenges Towards EU Harmonization. World Bank. (C) World Bank. https://openknowledge.worldbank.org/handle/10986/2754 License: Creative Commons Attribution CC BY 3.0.” 